\title{
Rhythm contour drives musical memory
}

\author{
Mark A. Schmuckler ${ }^{1 \dagger}$ \\ Rebecca Moranis ${ }^{2}$ \\ ${ }^{1}$ University of Toronto Scarborough, Toronto, ON, Canada \\ ${ }^{2}$ University of Toronto, Toronto, ON, Canada \\ † Corresponding author: mark.schmuckler@utoronto.ca \\ Published 16 December 2021; https://doi.org/10.18061/FDMC.2021.0045 \\ Author video presentation and/or other conference material: https://doi.org/10.17605/OSF.IO/M3RG5
}

\begin{abstract}
Two experiments examined listeners' use of contour information to drive memory for rhythmic patterns; these experiments were distinguished by the use of metric rhythms (Experiment 1) and ametric rhythms (Experiment 2). Both experiments employed a typical short-term memory task in which listeners heard a standard rhythm followed by a comparison rhythm. Comparison rhythms could be one of three types: an exact repetition of the standard rhythm, a same contour rhythm in which the relative durations of successive notes were comparable to the standard, and a different contour rhythm in which the relative durations of successive notes were modified relative to the standard. Analyses of $d$ primes for same/different detection revealed that, for both studies, listeners performed better when the comparisons had different rhythm contours, relative to comparisons with the same rhythm contours. These findings converge with results investigating melodic contour, and suggest that listeners both form and use contours of novel rhythmic patterns.
\end{abstract}

KEYWORDS: Rhythm, Contour, Short-term Memory, D primes

\section{Introduction}

Multiple authors have proposed that contour, along with tonality, are fundamental components of musical processing (Dowling, 1978; Schmuckler, 2016). These factors, to varying degrees, drive online processing of musical information, including perceptual organization (Bregman, 1990; Krumhansl \& Schmuckler, 1986). complexity (Eerola et al., 2006; Schmuckler, 1999), similarity (Prince, 2014; Schmuckler, 2010), memory (Dowling \& Fujitani, 1971; Halpern \& Bartlett, 2010), and performance (Drake \& Palmer, 2000; Lewandowska \& Schmuckler, 2019). Given this wealth of evidence, it is clear that both of these components play central roles in all aspects of musical behavior.

One characteristic of tonality and contour is that the underlying structure of these components involves the organization of pitch information. Thus, work on tonality has been driven by investigation of the tonal hierarchy (Krumhansl, 1990, 2000; Krumhansl \&
Cuddy, 2010). Work on contour has similarly focused on characterizing models of pitch structure (Adams, 1976; Friedmann, 1985; Marvin \& Laprade, 1987; Quinn, 1999; Schmuckler, 1999). Other work has extended the formal characterizations of tonality (i.e., its hierarchical structure) to other musical dimensions, such as meter and rhythm (Martin, 1972; Povel \& Essens, 1985; Povel, 1981, 1984). In contrast, although the concept of contour is discussed relative to other perceptible dimensions, most notably vision (e.g., Koenderink et al., 1997), it has only rarely been discussed with reference to other auditory and/or musical dimensions (but see Schmuckler \& Gilden, 1993, for one notable exception).

Given this paucity of previous work, what might be an appropriate context for investigating the formation and use of contour in the temporal domain? Methodologically, it would make sense to employ paradigms that have been used successfully to investigate perceived contour in other musical domains. Within this framework, it is most instructive to look at work investigating melodic contour. Specifically, work by Dowling and colleagues (e.g., Bartlett \& Dowling, 1980; Dowling, 1978, 1994; Dowling \& Bartlett, 1981; Dowling \& Fujitani, 1971; Dowling et al., 1995; Halpern \& Bartlett, 2010; Halpern et al., 1998) provides some of the most comprehensive and well-known research on melodic contour. This work has demonstrated that listeners make use of similar contour codes for both short- and long-term memory of musical melodies, although contour information is more critical for short-term melodic representations, and is enhanced when the melodies adhere to a coherent tonal framework (Dowling et al., 1995).

In Dowling's work, melodic contour is coded as a series of +'s and -'s, representing relative pitch differences between successive notes; this general form of contour coding (as well as its equivalent of 1's and -1's) has been employed by multiple authors (Friedmann, 1985; Marvin \& Laprade, 1987; Quinn, 
1999). Unfortunately, little work exists on how to characterize duration and/or rhythmic contours, with the majority of work analyzing rhythm more focused on patterns of stress and intonation, as opposed to durations, likely due to the emphasis on rhythm and prosody in speech and language (e.g., Aiello, 1994; Cooper \& Meyer, 1960; Lerdahl \& Jackendoff, 1983; Patel et al., 2006; Thaut, 2008). Cooper and Meyer's (1960) classic text on the rhythmic structure of music, for instance, explicitly relates musical rhythmic structure to accented and unaccented groupings, using terminology drawn from work in prosody.

Marvin (1991) proposed a characterization of rhythmic contours more consistent with the framework employed in Dowling's research. Specifically, Marvin suggests encoding rhythm contours "as analogous to melodic contours: they represent relative durations in much the same way that melodic contours represent relative pitch height, without a precise calibration of the intervals spanned" (Marvin, 1991, p. 64).

Based on this framework, one means for investigating whether contour plays a role in processing the temporal/rhythmic dimension in music thus makes use of the classic melodic contour paradigm pioneered by Dowling and colleagues (e.g., Dowling, 1984; Dowling et al., 2008). Although this research has employed a variety of paradigm variants in its explorations, one of the most basic procedures involves simply presenting an initial standard melody, followed (after a short interval) by a subsequent test melody, and asking participants if these two melodies were the same or different (e.g., Dowling et al., 2008). As shown in Figure 1, the relation between the standard and comparison melody can take multiple forms, with the comparison being an exact pitch transposition of the standard melody (Figure 1b), a same contour, but different pitch interval version of the standard (Figure 1c), and a different contour, and hence different pitch interval, version of the standard (Figure 1d). Findings from multiple studies have revealed listeners' accuracy in determining the relation between standard and comparison is driven by contour similarity, with listeners correctly discriminating melodies with different contours, but failing to discriminate melodies with the same contour. Interestingly, these findings hold for both tonal (Dowling, 1978) and atonal (Dowling \& Fujitani, 1971) melodies. The goal of the current study was to adapt this general framework for the perception of rhythmic contours, both within a metrical context (Experiment 1) and without a metrical framework (Experiment 2).
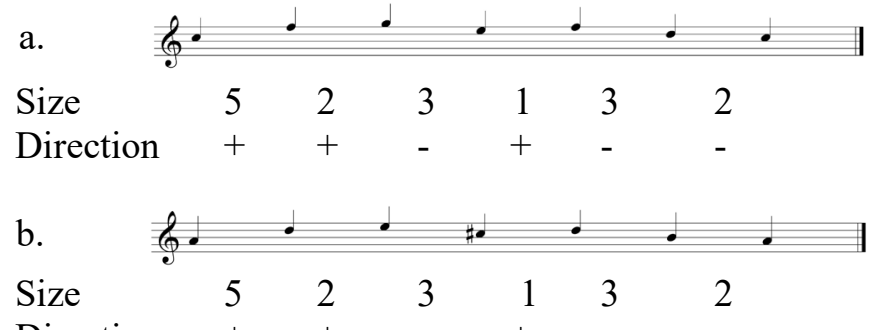
Direction $++\quad+\quad-\quad+$


Direction - $\quad-\quad+\quad+\quad-\quad$ -

Figure 1: Sample melodic contours, adapted from Dowling (1994). Figure la shows the standard contour, separating out pitch interval and direction information. Figure $1 b$ shows a comparison contour that is pitch transposed having the same pitch interval and direction information. Figure 1c shows a comparison contour having different pitch interval information, but the same pitch direction information. Figure $1 d$ shows a comparison contour with different pitch interval and direction information. For all melodies, "size" refers to the number of semitones between notes, and "direction" indicates whether successive tones are higher (+) versus lower (-) in pitch.

\section{Method}

\section{Participants}

Participants were drawn from the Introductory Psychology subject pool at the University of Toronto Scarborough; participants were not selected based on their prior musical training. Overall, data from 20 participants in each of Experiments 1 and 2 were employed in this study. One additional participant in Experiment 1 completed the study, but this participant's data was removed for a failure to appropriately use the rating scale.

\section{Experimental Apparatus and Stimuli}

Stimuli for these studies consisted of either 8 note (Experiment 1) or 7 note (Experiment 2) rhythms, played using a piano sound, on a single tone $\left(\mathrm{F}_{4}\right)$. For the metric stimuli of Experiment 1, the meter was 
established by sounding 8 beats of a woodblock prior to the start of the rhythm, which was spread out over 8 subsequent beats. For the ametric stimuli of Experiment 2, no a priori framework was established, and the rhythms themselves occurred across variable timeframes, depending on the specific duration values of their component tones. For the ametric stimuli, the lengths of the standard, same, and different contours were roughly equivalent.

For both metric and ametric stimuli, a set of standard rhythmic contours were created. These standard contours then generated three comparison contours; these different comparisons appear in Figure 2a (metric rhythms) and Figure $2 \mathrm{~b}$ (ametric rhythms). The first comparison rhythm represents an exact repetition of the standard rhythm, played at a faster overall tempo (150 beats/min) than the standard (120 beats/min). The second comparison rhythm represents a same contour variant of the standard, again played at a faster overall tempo (150 beats/min). As seen in Figure 2 , the relative pattern of durations for this rhythm is equivalent to the pattern of durations in the standard. The third comparison rhythm represents a different contour variant of the standard, played at a faster overall tempo (150 beats/min), with the relative durations displaying a different pattern than the standard. Twenty different sets of standard and comparison rhythm contours were created for each of the metric and ametric experiments.

All listeners heard 80 trials of randomly ordered standard-comparison rhythm contour pairs. These pairs consisted of two repetitions of the exact repetition comparison, and one repetition each of the same contour and different contour comparisons, for each of the 20 rhythms. The two repetitions of the exact repetition were included to balance the number of objective "same" and "different" responses. Listeners were instructed to judge whether standard and comparison rhythms were exactly the same (ignoring the overall tempo). They made these judgments using a $1-6$ scale, with responses $1-3$ indicating the rhythms were different at varying levels of confidence ( 1 = very confident; $3=$ mildly confident $)$, and responses $4-6$ indicating the rhythms were the same at varying levels of confidence $(4=$ mildly confident; 6 = very confident).

\section{Results}

For each listener, the 6-point rating scale was used to calculate percent values (i.e., responding $1-3$ for same contour and different contour comparisons, and $4-6$ for the exact repetition contours). These percent values were then used to calculate $d$ prime scores separately for the same contour and different contour rhythms, using the percent correct for these two stimuli as their respective hit rate, and 1-percent correct for the exact repetition as the false alarm rate.

\section{a. Metric Rhythms (Experiment 1)}
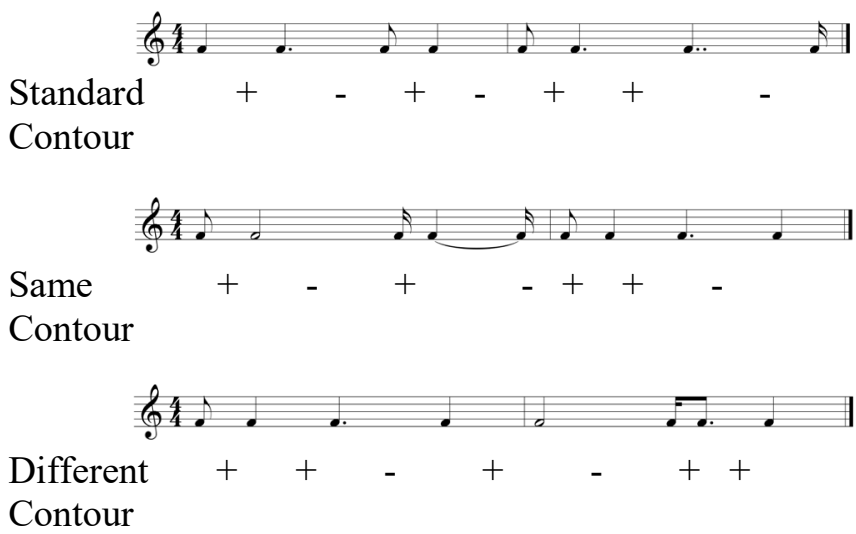

b. Ametric Rhythms (Experiment 2)

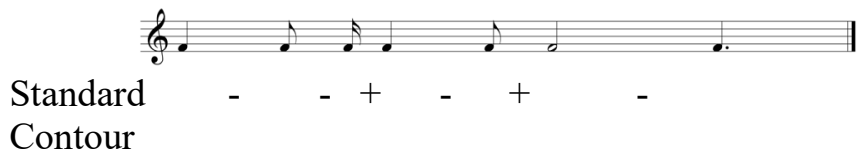

Same

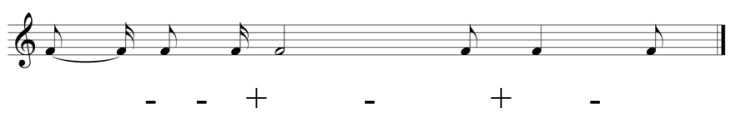

Contour

Different

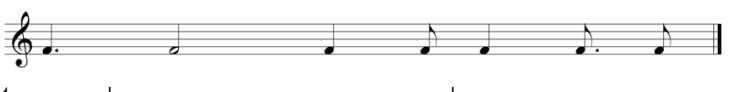

Contour

Figure 2: Standard, same, and different contours for the metric rhythms of Experiment 1 (a) and ametric rhythms of Experiment 2 (b). Increases in relative durations between notes are indicated with a "+", and decreases in relative durations are indicated with a "-".

D prime scores for metric (Experiment 1) and ametric (Experiment 2) rhythms were analyzed in oneway ANOVAs, with the within subjects factor of Contour Type (same contour, different contour); d prime measures incorporate the hit rate $(\%$ correct for exact repetitions) into the overall measure. For the metric stimuli, this analysis produced a main effect of Contour Type, $F(1,19)=5.74, M S E=0.266, p<.05, n p^{2}=.232$. 
Mean values for this analysis appear in Figure 3a. For the ametric stimuli, this analysis also revealed a main effect of Contour Type, $F(1,19)=18.04, M S E=0.072$, $p<.001, n p^{2}=.487$. Figure 3 b shows the mean values for this analysis. a. Metric Rhythms

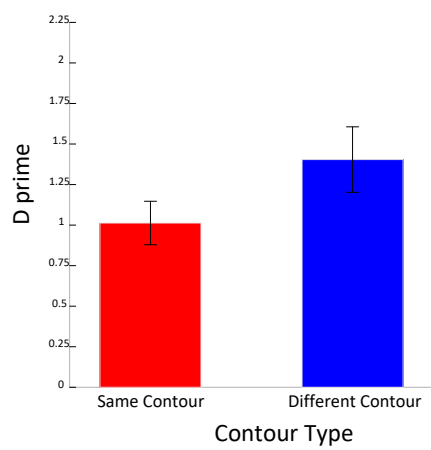

b. Ametric Rhythms

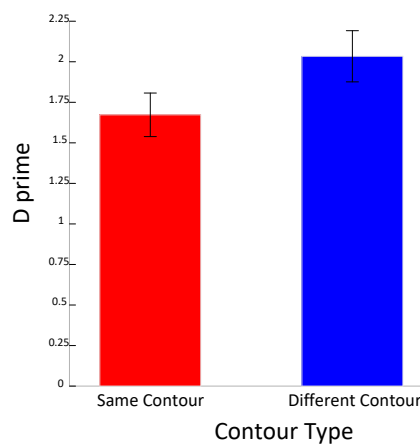

Figure 3: D primes (and SEs) for discrimination of same, and different contours for the metric rhythms of Experiment 1 (a) and ametric rhythms Experiment 2 (b).

\section{Discussion}

Two experiments investigated listeners' use of contour information in short-term memory for rhythmic patterns. These studies found that contour similarity led to memory confusions, such that listeners were less able to discriminate a new rhythmic pattern from an old pattern when the two rhythms shared the same pattern of relative durations. Thus, these findings suggest that listeners are able to form duration contours, and that when hearing a rhythm, listeners are encoding the relative patterns of durations, as opposed to the actual durations of the rhythms. This process, in many ways, is comparable to that observed in the processing of melodic contour, in which listeners encode the relative patterns of pitch changes, as opposed to the exact pattern of pitch intervals (Dowling, 1994).

Somewhat more surprisingly, these studies found little difference in rhythm encoding as a function of whether or not the underlying rhythm was set in a metric framework. Comparison of Experiments 1 and 2 showed that both metric and ametric rhythms produced equivalent influences on memory of same versus different contours. Although intuitively surprising, this finding converges with results from the melodic contour literature in which contour information plays a role in melodic memory for both tonal (Dowling, 1978) and atonal (Dowling \& Fujitani, 1971) melodies. Future work might profitably explore these results by investigating the impact of contour durations on pitch and rhythm processing simultaneously, as well as looking at factors such as short- versus long-term memory, which influences the relative impact of tonal and interval information.

\section{Acknowledgements}

This research was supported by a grant from the Natural Sciences and Engineering Research Council to Mark A. Schmuckler.

\section{References}

Adams, C. R. (1976). Melodic contour typology. Ethonomusicology, 20, 179-215. https://doi.org/10.2307/851015

Aiello, R. (1994). Music and language: Parallels and contrasts. In R. Aiello (Ed.), Musical perceptions (pp. 4063). Oxford University Press.

Bartlett, J. C., \& Dowling, W. J. (1980). Recognition of transposed melodies: A key-distance effect in developmental perspective. Journal of Experimental Psychology: Human Perception and Performance, 6, 501-515. https://doi.org/10.1037/0096-1523.6.3.501

Bregman, A. S. (1990). Auditory scene analysis: The perceptual organization of sound. The MIT Press. https://doi.org/10.7551/mitpress/1486.001.0001

Cooper, G., \& Meyer, L. B. (1960). The rhythmic structure of music. Chicago University Press.

Dowling, W. J. (1978). Scale and contour: Two components of a theory of memory for melodies. Psychological Review, 85, 341-354. https://doi.org/10.1037/0033295X.85.4.341

Dowling, W. J. (1984). Musical experience and tonal scales in the recognition of octave-scrambled melodies. Psychomusicology, 4, 13-32. https://doi.org/10.1037/h0094206

Dowling, W. J. (1994). Melodic contour in hearing and remembering melodies. In R. Aiello \& J. Sloboda (Eds.), Musical Perceptions (pp. 173-190). Oxford University Press.

Dowling, W. J., \& Bartlett, J. C. (1981). The importance of interval information in long-term memory for melodies. Psychomusicology, 1, 30-49. https://doi.org/10.1037/h0094275

Dowling, W. J., Bartlett, J. C., Halpern, A. R., \& Andrews, M. W. (2008). Melody recognition at fast and slow tempos: Effects of age, experience, and familiarity. Perception and Psychophysics, 70, 496-502. https://doi.org/10.3758/PP.70.3.496 
Dowling, W. J., \& Fujitani, D. S., (1971). Contour, interval and pitch recognition in memory for melodies. Journal of the Acoustical Society of America, 49, 524-531. https://doi.org/10.1121/1.1912382

Dowling, W. J., Kwak, S., \& Andrews, M. W. (1995). The time course of recognition of novel melodies. Perception and Psychophysics, 57, 150-158. https://doi.org/10.3758/BF03206500

Drake, C., \& Palmer, C. (2000). Skill acquisition in music performance: Relations between planning and temporal control. Cognition, 74, 1-32. https://doi.org/10.1016/S0010-0277(99)00061-X

Eerola, T., Himberg, T., Toiviainen, P., \& Louhivuori, J. (2006). Perceived complexity of western and African folk melodies by western and African listeners. Psychology of Music, 34, 337-371. https://doi.org/10.1177/0305735606064842

Friedmann, M. L. (1985). A methodology for the discussion of contour: Its application to Schoenberg's music. Journal of Music Theory, 29, 223-248. https://doi.org/10.2307/843614

Halpern, A. R., \& Bartlett, J. C. (2010). Memory for melodies. In M. R. Jones, R. R. Fay, \& A. N. Popper (Eds.), Music Perception (pp. 233-258). SpringerVerlag. https://doi.org/10.1007/978-1-4419-6114-3_8

Halpern, A. R., Bartlett, J. C., \& Dowling, W. J. (1998). Perception of mode, rhythm and contour in unfamiliar melodies: Effects of age and experience. Music Perception, 15, 335-355. https://doi.org/10.2307/40300862

Koenderink, J. J., van Doorn, A. J., Kappers, A. M. L., \& Todd, J. T. (1997). The visual contour in depth. Perception and Psychophysics, 59, 828-838. https://doi.org/10.3758/BF03205501

Krumhansl, C. L. (1990). Cognitive foundations of musical pitch. Oxford University Press.

Krumhansl, C. L. (2000). Rhythm and pitch in music cognition. Psychological Bulletin, 126, 159-179. https://doi.org/10.1037/0033-2909.126.1.159

Krumhansl, C. L., \& Cuddy, L. L. (2010). A theory of tonal hierarchies in music. In M. R. Jones, R. R. Fay, \& A. N. Popper (Eds.), Music Perception (pp. 51-87). Springer. https://doi.org/10.1007/978-1-4419-6114-3_3

Krumhansl, C. L., \& Schmuckler, M. A. (1986). The Petroushka chord: A perceptual investigation. Music Perception, 4, 153-184. https://doi.org/10.2307/40285359

Lerdahl, F., \& Jackendoff, R. (1983). A generative theory of tonal music. MIT Press.

Lewandowska, O. P., \& Schmuckler, M. A. (2019). Tonal and textural influences on musical sight-reading. Psychological Research, 84, 1920-1945. https://doi.org/10.1007/s00426-019-01187-1
Martin, J. (1972). Rhythmic (hierarchical) vs. serial structure in speech and other behavior. Psychological Review, 79, 487-509. https://doi.org/10.1037/h0033467

Marvin, E. W. (1991). The perception of rhythm in nontonal music: Rhythmic contours in the music of Edgard Varèse. Music Theory Spectrum, 13, 61-78. https://doi.org/10.2307/745974

Marvin, E. W., \& Laprade, P. A. (1987). Relating musical contours: Extensions of a theory for contour. Journal of Music Theory, 31, 225-267. https://doi.org/10.2307/843709

Patel, A. D., Iversen, J. R., \& Rosenberg, J. C. (2006). Comparing the rhythm and melody of speech and music: The case of British English and French. The Journal of the Acoustical Society of America, 119, 3034-3047. https://doi.org/10.1121/1.2179657

Povel, D.-J., \& Essens, P. J. (1985). Perception of temporal patterns. Music Perception, 2, 411-440. https://doi.org/10.2307/40285311

Povel, D. J. (1981). Internal representation of simple temporal patterns. Journal of Experimental Psychology: Human Perception and Performance, 7, 3-18. https://doi.org/10.1037/0096-1523.7.1.3

Povel, D. J. (1984). A theoretical framework for rhythm perception. Psychological Research, 45, 315-337. https://doi.org/10.1007/BF00309709

Prince, J. B. (2014). Contributions of pitch contour, tonality, rhythm, and meter to melodic similarity. Journal of Experimental Psychology: Human Perception and Performance, 40, 2319-2337. https://doi.org/10.1037/a0038010

Quinn, I. (1999). The combinatorial model of pitch contour. Music Perception, 16, 439-456. https://doi.org/10.2307/40285803

Schmuckler, M. A. (1999). Testing models of melodic contour similarity. Music Perception, 16, 295-326. https://doi.org/10.2307/40285795

Schmuckler, M. A. (2010). Melodic contour similarity using folk melodies. Music Perception, 28, 169-193. https://doi.org/10.1525/mp.2010.28.2.169

Schmuckler, M. A. (2016). Tonality and contour in melodic processing. In S. Hallam, I. Cross, \& M. Thaut (Eds.), The Oxford handbook of music psychology (2nd ed., pp. 143165). Oxford University Press. https://doi.org/10.1093/oxfordhb/9780198722946.013.1 4

Schmuckler, M. A., \& Gilden, D. L. (1993). Auditory perception of fractal contours. Journal of Experimental Psychology: Human Perception and Performance, 19, 641-660. https://doi.org/10.1037/0096-1523.19.3.641

Thaut, M. H. (2008). Rhythm, music, and the brain: scientific foundations and clinical applications. Routledge. 